\title{
Enhanced Artificial Bee Colony Algorithm for Travelling Salesman Problem using Crossover and Mutation
}

\author{
Vani Maheshwari \\ Maharana Pratap College of Technology, Gwalior \\ (M.P.)-India
}

\begin{abstract}
Swarm intelligence systems are made up of a population of simple agents interacting locally with each another and with their environment. Artificial bee colony (ABC) algorithm, particle swarm optimization (PSO), ant colony optimization (ACO), differential evolution (DE) etc, are some example of swarm intelligence. In this work, an efficient modified version of $\mathrm{ABC}$ algorithm is proposed, where two additional operator crossover and mutation operator is used in the $\mathrm{ABC}$ algorithm. Here Crossover operator is used after the employed bee phase and mutation operator is used after scout bee phase of $\mathrm{ABC}$ algorithm. Proposed algorithm is applied at standard travelling salesman problem (TSP) for checking the efficiency of proposed algorithm and also simulated results are compared with $\mathrm{ABC}$ with uniform mutation algorithm and Basic ABC algorithm. The simulated result showed that the proposed algorithm is better than all the modified version of ABC algorithm.
\end{abstract}

\section{KEYWORDS}

Artificial Bee Colony, crossover, Mutation, Genetic Algorithm, Travelling salesman problem.

\section{INTRODUCTION}

The problem of optimization is the most crucial problem in today's era and a great many work have been done to solve it. Previously a lot of work has been done on GA, ABC and hybridization of various evolutionary algorithms. There are few literatures available which compares their performance evaluation and suggests the best technique to be opted for specific problems.

Karaboga proposed the $\mathrm{ABC}$ algorithm based on a particular intelligent behavior of the honeybee swarms $[1,2,3]$. In this work, a particular intelligent behavior of a honey bee swarm, foraging behavior, is considered and a new artificial bee colony $(\mathrm{ABC})$ algorithm simulating this behavior of real honey bees is described for solving multidimensional and multimodal optimization problems. A novel hybrid swarm intelligent approach is proposed by integrating both $\mathrm{ABC}$ and GA with introducing two information exchange processes between GA population and bee colony [4]. In [5], a crossover step is added to the standard PSO. The crossover operation is used for each offsprings for individual best offspring. After the crossover, the fitness value of the individual best position is compared with that of the two chromosomes, and the best offspring is taken as the new individual best position. In paper $[6,7]$, Many studies are available in which PSO and crossover operators are applied. Genetic algorithms (GAs) are a family of computational models developed by Holland [8, 9, 10], which is based on the principles of natural biological evolution.

The organization of the paper is as follows: section 2 gives different modified version of Artificial Bee Colony algorithm.

\author{
Unmukh Dutta \\ Maharana Pratap College of Technology, Gwalior
}

(M.P.)-India

Section 3 gives travelling salesman problem, Section 4 explains proposed methodology. Section 5 describes the Experimental results and parameter setup for experiments and Section 6 concludes the work.

\section{DIFFERENT MODIFIED VERSION OF ABC ALGORITHMS}

\subsection{ABC Algorithm with Uniform Mutation}

In this Work [11], one additional step, i.e. mutation operator, is added to standard artificial bee colony algorithm. Mutation operator is added after the employed bee phase of $\mathrm{ABC}$ algorithm. Artificial Bee Colony algorithm has four phases: initialization bee phase, employed bees phase, onlooker bees phase and scout bees phase. Employed bee phase do the local search and mutation after the employed bee phase explore the search space and do the searching new area of solution space. By using the mutation operator, at the one side, there is a probability of changing the temporary best position, and the algorithm may not be depend on local solution. On the other side, individual can make use of the others' advantage by sharing information mechanism. In this method, the mutation step is carried out on the probabilistic way in each food searching operation for each iteration during the life cycle of $\mathrm{ABC}$ optimization technique. Selection of food source is done in a random manner. Food Source is selected randomly from the food size and mutation operation is performed. In mutation, generated offspring's replaces the older offspring's. in this work, uniform mutation is used. When performing mutation, we randomly select one food source and replace its one of the dimension value by random number generated in between lower and upper bound value of the food source.

\subsection{Artificial Bee Colony Algorithm}

In the Artificial Bee Colony algorithm, there are three different phases of Artificial Bee Colony algorithm: employed bee, onlooker bee and scout bee phase. The natural behavior of bee waiting on the dance area for taking decision to choose a food source, is called an onlooker bee phase of $\mathrm{ABC}$ algorithm and a bee going to the food source moved by itself previously is named an employed bee phase of $\mathrm{ABC}$ algorithm. A bee having the randomized search is known as a scout bee phase. In the Artificial Bee Colony algorithm, first half of the bee colony contain of employed artificial bees and the second half contain the onlooker bee. For every food source, there is only single employed bee. In other words, the numbers of employed bees are same as the number of food sources surrounded the hive. The employed bee whose food source is randomized by the employed and onlooker bees becomes a scout bee. Followings are the main steps of the algorithm: 
Step 1: Initialize.

\section{Step 2: REPEAT}

a) Place employed bees on the food sources in the memory;

b) Place onlooker bees on the food sources in the memory;

c) Send scouts to the search area for discovering new food sources.

Step 3: UNTIL (Requirements are met).

\section{TRAVELLING SALESMAN PROBLEM}

The Traveling Salesman Problem (TSP) is one of the optimization problem which have been widely used extensively by computer scientists and mathematicians. Travelling Salesman Problem is an example of combinatorial optimization problem, known to be the NP-complete problem. It naturally arises as a sub-problem in various application areas such as network communication, manufacturing and transportation [13]. Formally, the TSP may be defined as follows [14], it is a permutation problem with the objective of finding the path of the minimum length (or the minimum cost) on an undirected graph that represents cities or nodes to be visited. The traveling salesman starts from one node or city, visits all other nodes successively only one time each node, and finally returns to the starting node. That is, given $\mathrm{n}$ cities $\left(c_{1}, c_{2} \ldots c_{n}\right)$ and permutations $\left(\sigma_{1} \ldots \sigma_{n}\right)$. The objective is to choose $\sigma_{i}$ such that the sum of all Euclidean distances between each node and its successor is minimized. Successor of the last node in the permutation is the first one node. Euclidean distance $\mathrm{d}$, between any two nodes with coordinate $\left(\mathrm{x}_{1}, \mathrm{y}_{1}\right)$ of one node and $\left(\mathrm{x}_{2}, \mathrm{y}_{2}\right)$ of second node is calculated by

$$
\mathrm{d}=\sqrt{(x 1-x 2)^{2}+(y 1-y 2)^{2}}
$$

Generally, the Travelling Salesman Problem states that for one salesman who wants to visit $\mathrm{n}$ different cities, his objective would be to find the proper sequence of tour that minimizes the cost of travel by visiting each city or node exactly once and finally rests to the starting node. Travelling Salesman Problem is so easy to describe and so difficult to solve.

\section{PROPOSED METHODOLOGY}

In this proposed method, two additional steps are added to standard Artificial Bee Colony Optimization is of crossover and mutation operator. The first step of the $\mathrm{ABC}$ algorithm is to generate the population. Initial populations generated by $\mathrm{ABC}$ are used by employed bees. After this phase, crossover operators are applied. If crossover criteria or probability satisfies than two parents are selected randomly to perform crossover operation on them. New offsprings are generated after crossover operation. With best generated offspring, replace the worst offspring parent is done if it is better than the worst parent in terms of their fitness value. Here crossover operator is applied upon two randomized selected parents from the current population. Two offspring generated from crossover and replaced worst parent by best offspring, other parent remains same. Now mutation operator is applied after the scout bee phase of $\mathrm{ABC}$ algorithm. By using the mutation operator, on the one side, there is a chance of changing the local best position, and the algorithm may not be depend on local solution. On the other side, particle can make use of the others' advantage by sharing information mechanism. In this proposed method, the mutation step is carried out on the probabilistic way in each food searching operation for each iteration during the life cycle of $A B C$ optimization algorithm. Selection of food source is done in a randomized manner. Food Source is selected randomly from the food size and mutation is performed. In mutation, generated offspring's replaces the older offspring's. mutation operator used in this work is uniform mutation. When performing mutation, we randomly select one food source and replace its one of the dimension value by random number generated in between lower and upper bound value of the food source. Proposed algorithm is discussed below -

\section{Algorithm 1: ABC with Crossover and Mutation Operator} Step 1 : [Initialsation Phase]

for $\mathbf{i}=\mathbf{0}$ to the $\max$ no. of food source do

for $\mathbf{d}=\mathbf{0}$ to the dimension size $\mathbf{d o}$

Randomly initialize the food source positions Xij

end for $d$

Compute the fitness value of each food source

end for $i$

Repeat

Step 2 : [Employed Bee Phase]

for $\mathbf{i}=\mathbf{0}$ to max no of the employed bee do

for $\mathbf{d}=\mathbf{0}$ to dimension $\mathbf{d o}$

produce the new candidate solution

end for $d$

Compute the fitness value of individual

if the fitness value of new candidate solution is better than the existing solution replace the older solution.

end for $i$

for $\mathbf{i}=\mathbf{0}$ to the max no. of food source do

Calculate the probability for each food source.

end for $i$

Step 3 : [Crossover phase]

If crossover criteria satisfies

For $\mathrm{i}=0$ to the maximum no. of food source

Select any two random individuals from the current population for the crossover operation.

Apply crossover operation

New offspring generated from parents as a result of crossover operation. Replace the worst parent with the best new offspring if it is better.

End of $i$

Step 4 : [Onlooker Bee Phase]

for $\mathbf{i}=\mathbf{0}$ to the max no of onlooker bee do on the basis of probability $\mathrm{Pi}$, choose food source

for $\mathbf{d}=\mathbf{0}$ to dimension $\mathbf{d o}$

produce the new candidate solution for food source position Xij 


\section{end for $d$}

compute the fitness value of individual food source

if the fitness value of new candidate solution is better than the existing solution replace the older solution.

end for $i$

\section{Step 5: [Scout Bee Phase]}

If any food source exhausted than

replace it by the new random position generated by scout bee.

\section{Step 6: [Mutation Phase]}

If mutation criteria is met then

Select random particle from current population for mutation operator.

Apply mutation operation to generate new individuals new offspring generated from the result of mutation.

New set of sequence is produced for offspring

Compute cost for that offspring

Compute the fitness value of updated individual

Memorize best food source so far

Until (Stopping criteria met).

\section{EXPERIMENTAL RESULTS AND PARAMETER SETUP}

The first control Parameter is Maximum cycle number and the value of this parameter we have taken in our experiment as 2500 . The next parameter in our experiment is maximum number of food source and we have taken its value to be 30 . Another control parameter is number of runs and we have taken its value in our experiment as 30 . It must be noted that each run contains max. cycle number, which is taken as 2500 in our experiment. The fourth control parameter is Dimension, whose value taken as 30 . Next control parameter for mutation operator is Probability. Therefore we need to find the value of this parameter also. value of the mutation probability is 0.2 taken here.

For solving Travelling salesman problem by using proposed algorithm, we have taken the result for 500,1000 and 1500 MCN value respectively and the colony size equals to the population size, i.e. 20 . The percentage of onlooker bees was $50 \%$ of the colony, the employed bees were $50 \%$ of the colony and the number of scout bees was selected as one. The dimension of each individual is taken as 10, 20, and 30 respectively. Each of the experiments was repeated 30 times with different random seeds. For $\mathrm{ABC}, \mathrm{ABC}$ with uniform mutation and proposed $\mathrm{ABC}$ algorithm, mean cost function value of TSP were calculated and compared. Experimental results on different dimensions are shown in table 1 , table 2 and table 3 and graphical representation shown in figure 1, figure 2 and figure 3 respectively:
Table 1 Comparison results For Dimension $D=10$

\begin{tabular}{|c|c|c|c|}
\hline Algorithm & $\begin{array}{c}\text { Max } \\
\text { Cycle } \\
\mathbf{5 0 0}\end{array}$ & $\begin{array}{c}\text { Max } \\
\text { Cycle } \\
\mathbf{1 0 0 0}\end{array}$ & $\begin{array}{c}\text { Max } \\
\text { Cycle } \\
\mathbf{1 5 0 0}\end{array}$ \\
\hline ABC & 60.67 & 58.73 & 57.93 \\
\hline $\begin{array}{c}\text { ABC with Uniform } \\
\text { Mutation }\end{array}$ & 58.50 & 57.62 & 56.11 \\
\hline Proposed ABC & $\mathbf{5 6 . 4 5}$ & $\mathbf{5 6 . 1 2}$ & $\mathbf{5 5 . 2 8}$ \\
\hline
\end{tabular}

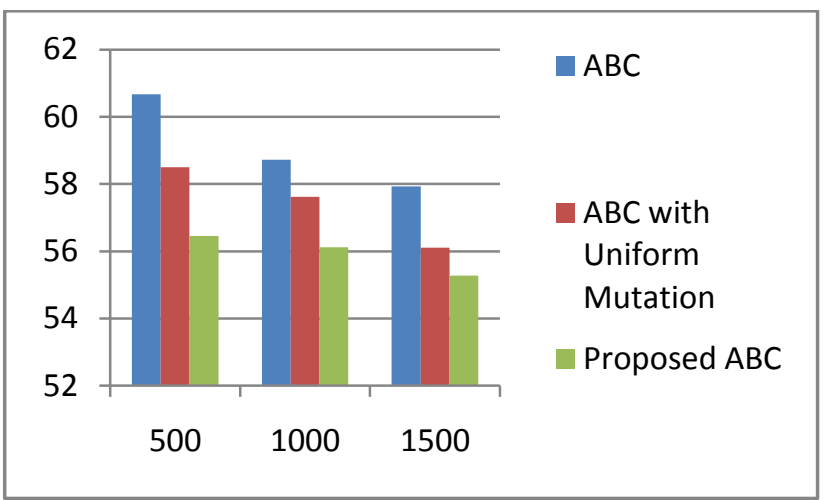

Fig 1 Comparative results at different $\mathrm{MCN}$ for $\mathrm{D}=10$

Table 2 Comparison results For Dimension D=20

\begin{tabular}{|c|c|c|c|}
\hline Algorithm & $\begin{array}{c}\text { Max } \\
\text { Cycle } \\
\mathbf{5 0 0}\end{array}$ & $\begin{array}{c}\text { Max } \\
\text { Cycle } \\
\mathbf{1 0 0 0}\end{array}$ & $\begin{array}{c}\text { Max } \\
\text { Cycle } \\
\mathbf{1 5 0 0}\end{array}$ \\
\hline ABC & 150.20 & 141.0 & 135.20 \\
\cline { 1 - 2 } ABC with Uniform \\
Mutation
\end{tabular}

Fig 2 Comparative results at different $M C N$ for $D=10$ 
Table 3 Comparison results For Dimension $D=30$

\begin{tabular}{|c|c|c|c|}
\hline Algorithm & $\begin{array}{c}\text { Max } \\
\text { Cycle } \\
500\end{array}$ & $\begin{array}{c}\text { Max } \\
\text { Cycle } \\
1000\end{array}$ & $\begin{array}{c}\text { Max } \\
\text { Cycle } \\
1500\end{array}$ \\
\hline $\mathrm{ABC}$ & 208.76 & 196.60 & 191.50 \\
\hline $\begin{array}{c}\text { ABC with Uniform } \\
\text { Mutation }\end{array}$ & 206.49 & 195.12 & 189.35 \\
\hline Proposed ABC & 204.36 & 194.90 & 188.20 \\
\hline \multicolumn{2}{|l|}{215} & \multirow{2}{*}{\multicolumn{2}{|c|}{$\mathrm{ABC}$}} \\
\hline \multicolumn{2}{|l|}{210} & & \\
\hline \multicolumn{4}{|l|}{205} \\
\hline \multicolumn{4}{|l|}{200} \\
\hline \multicolumn{4}{|c|}{$A B C$ with } \\
\hline \multicolumn{4}{|l|}{190} \\
\hline \multicolumn{4}{|c|}{ Mutation } \\
\hline \multicolumn{4}{|c|}{ Pronosed ABC } \\
\hline 500 & 1500 & & \\
\hline
\end{tabular}

Fig 3 Comparative results at different $\mathrm{MCN}$ for $\mathrm{D}=\mathbf{3 0}$

\section{CONCLUSION}

In this paper, real coded crossover and mutation operator is applied after the employed bee phase and scout bee phase of $\mathrm{ABC}$ algorithm. With the use of crossover operator, new offsprings are generated from initial population and replace the worst parent with best offspring and with mutation operator, randomly select one food source and replace its one of the dimension value by random number generated in between lower and upper bound value of the food source. The experiments are performed on Travelling Salesman Problem and obtained results are compared with simple $\mathrm{ABC}$ algorithm and $\mathrm{ABC}$ with Mutation Algorithm. As future work to apply proposed algorithm to other problems for checking the efficiency.

\section{REFERENCES}

[1] Dervis Karaboga - Bahriye Basturk, "A powerful and efficient algorithm for numerical function optimization: artificial bee colony (ABC) algorithm" J Glob Optim (2007), pp 459-471

[2] D. Karaboga, "An idea based on honey bee swarm for numerical optimization," Erciyes University, Engineering Faculty, Computer Engineering Department, Turkey, Technical Report-TR06, 2005

[3] Basturk, B., Karaboga, D. "An artificial bee colony $(\mathrm{ABC})$ algorithm for numeric function optimization". In:
IEEE Swarm Intelligence Symposium 2006, Indianapolis, Indiana, USA (May 2006).

[4] Xiaohu Shi, Yanwen Li, Haijun Li, Renchu Guan, Liupu Wang and Yanchun Liang: "A Hybrid Swarm Intelligent Method Based on Genetic Algorithm and Artificial Bee Colony," ICSI 2010, Part I, LNCS 6145, pp. 558-565, 2010. Springer-Verlag Berlin Heidelberg 2010.

[5] Zhi-Feng Hao, Zhi-Gang Wang and Han Huang, "A Particle swarm optimization algorithm with crossover operator", in Proc. of the Sixth International Conference on Machine Learning and Cybernetics, Hong Kong, 1922 August 2007.

[6] Dongyong Yang, Jinyin Chen and Matsumoto Naofumi, "Self-adaptive Crossover Particle Swarm Optimizer for Multi-dimension Functions Optimization”, ICNC 2007.

[7] Xie, Jiahua., Yang, Jie., "A Novel Crossover Operator for Particle Swarm Algorithm ", Machine Vision and Human-Machine Interface (MVHI), 2010 , IEEE Pages $161-164$.

[8] J.H. Holland, " Adaptation in Natural and Artificial System," The D.E. Goldberg, "Genetic Algorithms in Search, Optimization \& Machine Learning. Reading," MA: Addison-Wesley, 1989.

[9] J.H. Holland, "Adaptation in Natural and Artificial System, The University of Michigan Press", Ann Arbor, 1975.

[10] G-. G. Jin, S-. R. Joo, "A Study on a Real-Coded Genetic Algorithm," Journal of Control, Automation, and Systems Engineering, vol. 6, no. 4, pp. 268-274, April 2000.

[11] A. Singh, N. Gupta and A. Singhal," Artificial bee colony algorithm with uniform mutation", Proceedings of the International Conference on Soft Computing for Problem Solving (SocProS 2011), Volume 130, 2012, pp 503-511, December 20-22, 2011.

[12] M. Gupta and G. Sharma," An Efficient Modified Artificial Bee Colony Algorithm for Job scheduling problem", International Journal of Soft Computing and Engineering (IJSCE), ISSN: 2231-2307, Volume -1, Issue -6, January2012.

[13] L.-P. Wong, M.Y. Hea Low, C.S. Chong, "A Bee Colony Optimization Algorithm for Traveling Salesman Problem," Second Asia International Conference on Modeling \& Simulation, 2008, pp. 818-823.

[14] M. Lalena, "Traveling Salesman Problem Using Genetic Algorithms". http://www.lalena.com/ai/tsp/, 2003. 